\title{
3D-Printed Microfluidics and Potential Biomedical Applications
}

\author{
Priyanka Prabhakar ${ }^{1,2}$, Raj Kumar Sen ${ }^{1,2}$, Neeraj Dwivedi ${ }^{1,2 *}$, Raju Khan ${ }^{1,2}$, \\ Pratima R. Solanki ${ }^{3}$, Avanish Kumar Srivastava ${ }^{1,2}$ and Chetna Dhand ${ }^{1,2 *}$ \\ ${ }^{1}$ Industrial Waste Utilization, Nano and Biomaterials Division, CSIR-Advanced Materials and Processes Research Institute, \\ Bhopal, India, ${ }^{2}$ Academy of Scientific and Innovative Research (AcSIR), Ghaziabad, India, ${ }^{3}$ Special Centre for Nanoscience, \\ Jawaharlal Nehru University, New Delhi, India
}

3D printing is a smart additive manufacturing technique that allows the engineering of biomedical devices that are usually difficult to design using conventional methodologies such as machining or molding. Nowadays, 3D-printed microfluidics has gained enormous attention due to their various advantages including fast production, cost-effectiveness, and accurate designing of a range of products even geometrically complex devices. In this review, we focused on the recent significant findings in the field of 3D-printed microfluidic

OPEN ACCESS

Edited by: Anil Kumar, National Institute of Immunology (NII), India

Reviewed by: Rafal Walczak, Wrocław University of Science and Technology, Poland Prabhash Mishra, Moscow Institute of Physics and Technology, Russia

*Correspondence: Neeraj Dwived neerajdwivedi6@gmail.com Chetna Dhand chetnachem24@gmail.com

Specialty section: This article was submitted to Biomedical Nanotechnology, a section of the journal Frontiers in Nanotechnology

Received: 23 September 2020 Accepted: 25 January 2021 Published: 16 March 2021

Citation:

Prabhakar P, Sen RK, Dwivedi N, Khan R, Solanki PR, Srivastava AK and

Dhand C (2021) 3D-Printed

Microfluidics and Potential

Biomedical Applications.

Front. Nanotechnol. 3:609355. doi: 10.3389/fnano.2021.609355 devices for biomedical applications. 3D printers are used as fabrication tools for a broad variety of systems for a range of applications like diagnostic microfluidic chips to detect different analytes, for example, glucose, lactate, and glutamate and the biomarkers related to different clinically relevant diseases, for example, malaria, prostate cancer, and breast cancer. 3D printers can print various materials (inorganic and polymers) with varying density, strength, and chemical properties that provide users with a broad variety of strategic options. In this article, we have discussed potential 3D printing techniques for the fabrication of microfluidic devices that are suitable for biomedical applications. Emerging diagnostic technologies using 3D printing as a method for integrating living cells or biomaterials into 3D printing are also reviewed.

Keywords: 3D printing, microfluidics, biosensor, disease diagnosis, biomedical applications

\section{INTRODUCTION}

Microfluidics is the science and technology used in channels with a range of 10-100 micrometers to control small amounts of fluid $\left(10^{-9}\right.$ to $\left.10^{-18}\right)$. It offers new capabilities in space and time to control molecular concentrations (Tarn and Nicole, 2013; Bragheri et al., 2016). In terms of its bulk flow equivalent, microfluidic technology has several persuasive advantages, such as the need for fewer reagents and sample intake, favorable thermodynamics and chemical reaction kinetics, the profile of laminar flows, precise handling of single bioparticle, and strong parallels and multiplexing speeds (Knowlton et al., 2016; Kadimisetty et al., 2018a). Microfluidic chips allow accurate routing of fluid streams, but adequate sensing environments are needed for the external and internal stimuli (Han et al., 2019). Microfluidics is a lucrative sector that enables many biochemical and clinical applications such as cancer screening, engineering of microphysiological devices, medicinal research, and point-of-care diagnostics. Microfluidic systems are often complex, timeconsuming, and costly with the requirement of sophisticated clean room setup (Weisgrab et al., 2019). 3D printing has changed the field of microfluidics significantly and also created innovative microfluidic devices that are usually impossible to design employing other approaches. Threedimensional printing allows science and technology to be transformed by producing custom-made, 


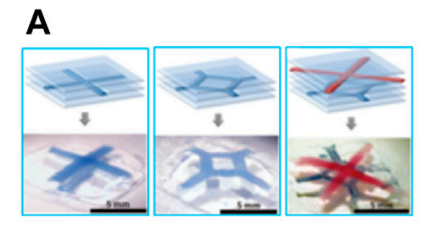

Three different designs of multi-

layered collagen scaffold with

fluidic channels and the actual constructions of those after gelatin removal using bioprinter

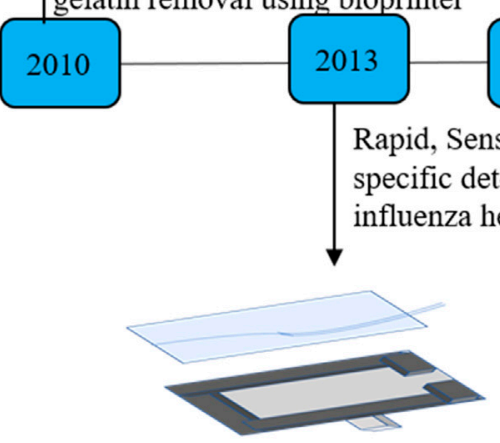

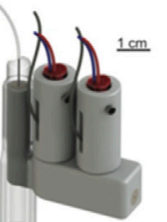

$\left[\begin{array}{l}\text { Biosensors that } \\ \text { measure glucose } \\ \text { and lactate level }\end{array}\right.$
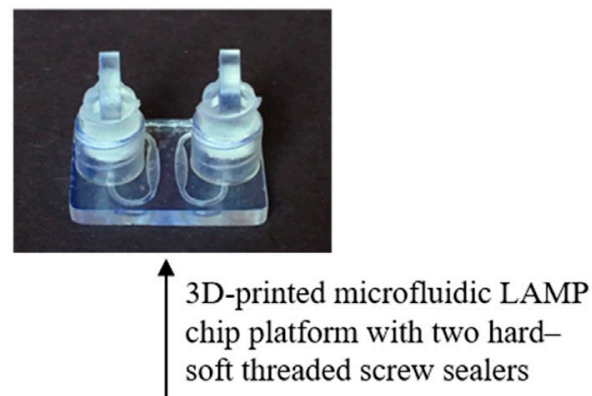

2015
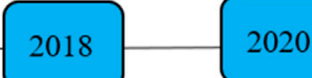

2020

$3 \mathrm{D}$ printed reactor

array for Nucleic acid amplification test

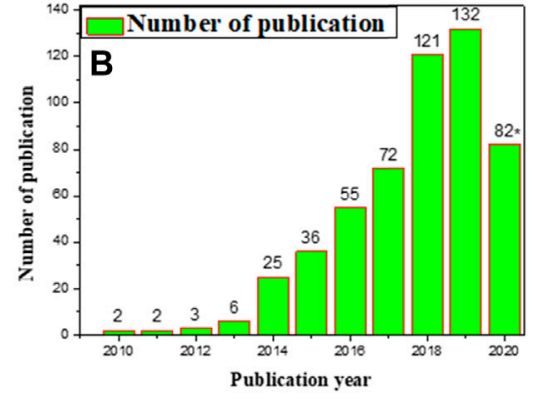

FIGURE 1 | (A) Timeline for producing 3D printing microfluidic devices in the last 10 years. Here, we emphasize on some examples of 3D printing microfluidic devices for biomedical applications. (B) The number of articles published on microfluidics and 3D printing annually. Data were obtained from "Web of Science" with "microfluidics and 3D printing" entered as "subject" in the search box (date: 07.09.2020). (Some portion of the figure is reproduced with permission of Lee et al., 2010; Gowers et al., 2015; Ruiz et al., 2020).

low-cost equipment requiring specialized equipment. An appealing but unexplored application is to use a $3 \mathrm{D}$ printer to initiate chemical reactions by printing the reagents directly into a $3 \mathrm{D}$ reaction ware matrix, putting reaction ware design, construction, and operation under digital control (Amin et al., 2016). 3D printing allows a microfluidic method but is currently limited to extraction-based printing and faces difficulties in applying it to other $3 \mathrm{D}$ printing modalities. 3D printing provides further potential for multi-material and multiphase printing (Symes et al., 2012). This article provides a detailed overview of 3D-printed microfluidics and their implication for different biomedical applications. One of the objectives of this review is to enrich the readers with knowledge about various potential 3D printing techniques explored for biomedical applications. Due to the main advantages of fast fabrication, simple accessibility, processing of different materials, and durability, 3D printing technology has flowered in sensing and for the development of 3D-printed microfluidic device for various applications in the biomedical sector (Zhang, 2019). Figure 1A shows the advancement in the development of 3D-printed microfluidic devices for various biomedical applications during the last 10 years. Figure 1B reveals the consistent enhancement in the total number of research publications in the field of 3D-printed microfluidics with progressing years.

\section{D PRINTING TECHNIQUES SUITABLE FOR BIOMEDICAL APPLICATION}

A variety of $3 \mathrm{D}$ printing techniques are available which are suitable for a wide range of applications. In this review, we will be focusing on and presenting the $3 \mathrm{D}$ printing techniques which are relevant to the biomedical field. Some of the techniques are discussed in the following text.

\section{Fused Deposition Modeling}

Fused deposition modeling is the most popular additive manufacturing technique for making 3D-printed devices for biomedical applications because of its ease and costeffectiveness. FDM is the extrusion-based $3 \mathrm{D}$ printing technique, in which thermoplastic polymeric materials are extruded to print objects layer by layer from a heated nozzle onto a surface or platform where it is cooled below its thermoplastic temperature, as shown in Figure 2A. This procedure is repeated until the $3 \mathrm{D}$ model is completed. FDM consists of the movable nozzle to print the materials in the $\mathrm{x}-\mathrm{y}$ direction through which the model is built layer by layer. FDM was widely used to print scaffolds that can be seeded with living cells without loss of cell viability and to print biofriendly polymer materials. Polylactic acid (PLA), nylon, acrylonitrile butadiene 


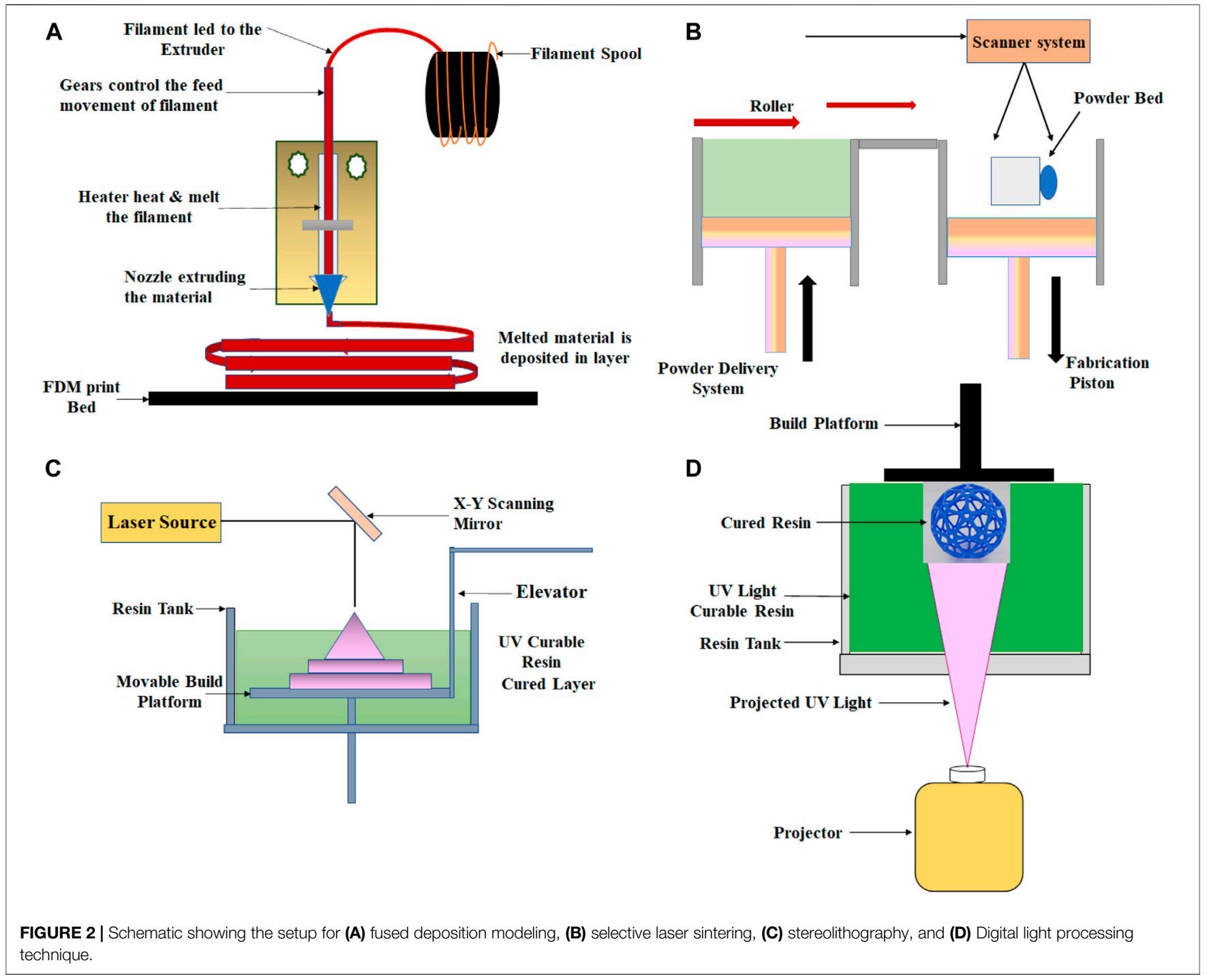

styrene (ABS), wax blend, etc. are the commonly used thermoplastic materials in the FDM technique (Mohamed et al., 2015; Singh et al., 2016; Hagedorn 2017).

\section{Selective Laser Sintering}

SLS is a $3 \mathrm{D}$ printing technique which belongs to the family of powder bed fusion. In this technique, a laser beam is generally used to build layered objects by melting and fusing the powder, as shown in Figure 2B. In SLS, powder fusion is accomplished utilizing various particle-binding mechanisms, that is, by chemical reactions, by solid-state sintering, or by absolute or partial melting. The controlled laser beam scanning is used in this method to sinter the powder by heating. In this technique, the resolution parameter is dependent on the particle size of the powder, scanning speed and spacing, laser strength, and quality of the powder. To fabricate the final $3 \mathrm{D}$ object, the process is repeated several times. In the SLS process, polymers such as polystyrenes (PS), thermoplastic elastomers (TPE), polyamide (PA), polyaryletherketones (PAEK), and polycaprolactone (PCL) are mainly used as laser sintering materials (Muzaffar et al., (2020); Munir et al., (2017); https:/www.3dhubs.com/knowledgebase/indroduction-sls-3d-printing/).

\section{Stereolithography}

This additive manufacturing process is part of the Vat photopolymerization family. In this process, the ultraviolet (UV) laser beam is used to produce the object layer by layer, as shown in Figure 2C. In the stereolithography technique, the materials used are photosensitive thermoset polymers in the liquid form, and the procedure is repeated until the object is eventually created. By controlling the direction of the UV laser beam, the polymerization of the resin can be controlled to achieve the desired structure and design. The main advantages of the SL technique are high surface resolution and precision. Using this method, high-resolution products can be produced while keeping the minimum cost due to the relatively restricted use of the liquid medium (Melchels et al., 2010; Salonitis, 2014; Ko et al., 2017). 


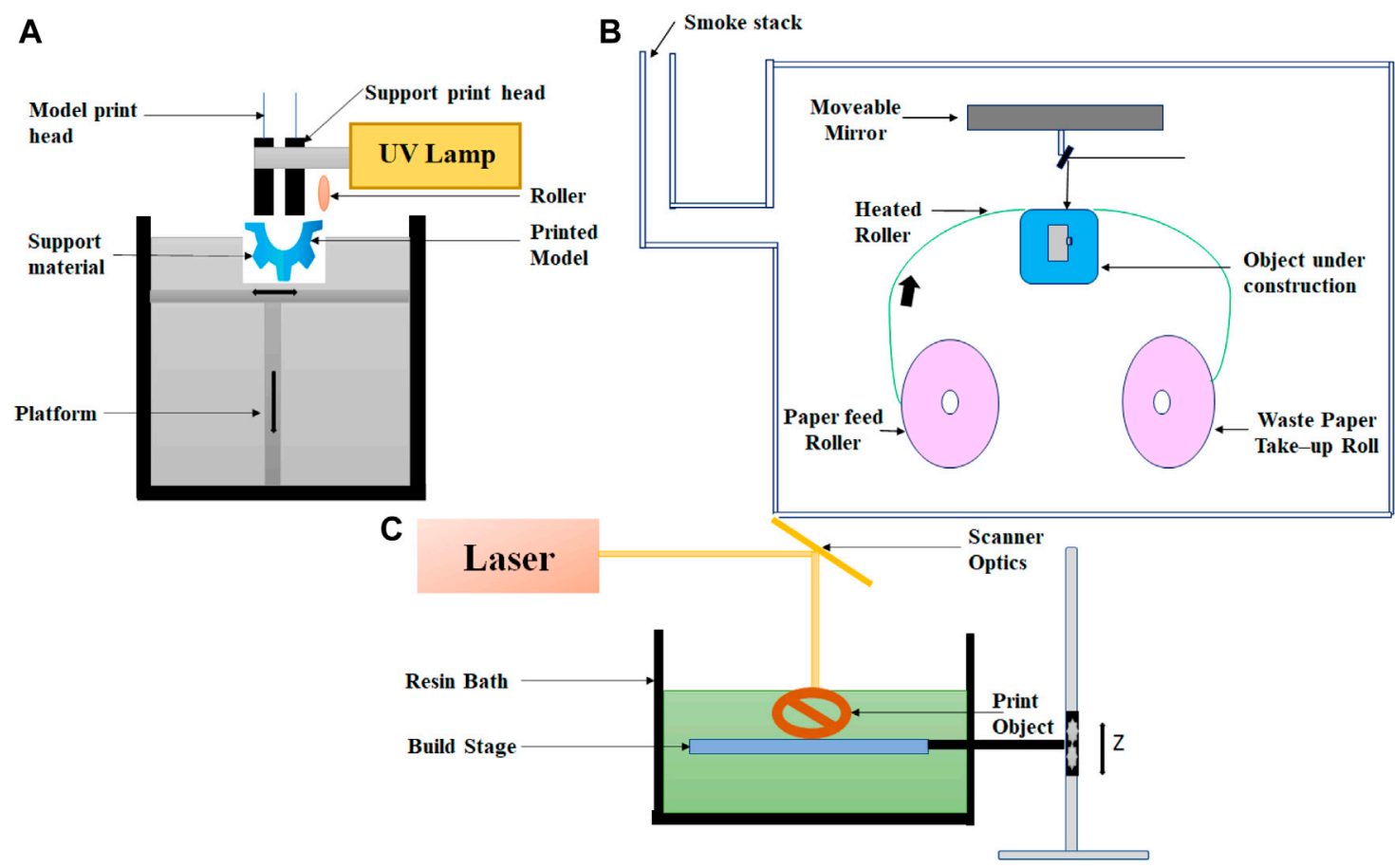

FIGURE 3 | Schematic for various 3D-printed techniques. (A) PolyJet 3D printing, (B) laminated object manufacturing, and (C) direct laser writing.

\section{Digital Light Processing}

DLP, an additive manufacturing technique, also belongs to the Vat photopolymerization family. DLP is quite similar to the SLA technique, except for the different photocuring process. In the DLP technique, the printer is used for the treatment of photopolymer resins, and UV light from the projector (light bulb) has been used to cure the photopolymer resin. In this process, each 2D layer is created after the liquid polymer is exposed to the light of the projector under the safest conditions, instead of having a layer with many laser scan paths, as shown in Figure 2D. DLP was used to build active component sensors that would alter their shape accordingly. In this technique, prototypes can be produced with good resolution, repeatability, and high accuracy (Schönberger and Hoffstetter, 2016; https://all3dp.com/ 2/what-is-a-dlp-3d-printer-3d-printing-simply-explain/).

\section{PolyJet Process}

The Polyjet process resembles inkjet printing. In this 3D printing technique, objects are built by jetting thousands of photopolymer droplets onto a built-in substrate and solidifying them with a UV light source, as shown in Figure 3A. The printer consists of many printing heads and movable platforms. Each printing head is filled with various types of liquid resins (different types of rubber or rigid, transparent, or opaque materials). In comparison to the inkjet process, a variety of materials may be sprayed and cured simultaneously. This technique has the potential to fabricate complex multi-material objects with smooth surface texture and with great accuracy. High-resolution objects of different modular strengths can be printed in three dimensions with high dimensional precision using this technology. Due to these capabilities, the PolyJet process is commonly used for different biomedical applications. (Tappa and Jammalamadaka, 2018; Matter-Parrat and Liverneaux, 2019; http://all3dp.com/2/ polyjet-3d-printing-technologies-simply-explained; https:// www.stratasys.co.in/polyjet-technology/).

\section{Laminated Object Manufacturing}

Laminated object manufacturing is one of the first commercially available $3 \mathrm{D}$ printing technique; it is based on layer-by-layer cutting and lamination of sheets or rolls of materials. A mechanical cutter or laser is used to cut the successive layer precisely, and then, it bonded together before it is laminated by the thermoplastic adhesive on top of the previous layer. Through a heated roller, the adhesive is activated, and the layer is laminated at a temperature between 60 and $80^{\circ} \mathrm{C}$ and a pressure of 10-30 MPa. Layers can be made out of metal sheets, plastic, or paper. The process may include postprocessing steps, including drilling and machining. The schematic diagram of LOM is shown in Figure 3B. This technique is very easy to use, cheap, and fast. Employing these techniques, relatively large-size objects can be fabricated but with a relatively lower resolution with the option of multicolor prototyping (Molitch-Hou, 2018; Ahangar et al., 2019; https://www.sculpteo.com/en/glossary/ lom-definition).

\section{Direct Laser Writing}

It is the type of laser-based 3D printing where the focused laser light is used to illuminate at a single focal point, either on the surface or within a volume of photopolymerizable materials; 
digitally controlled motorized stage and/or mirror galvanometers can then trace this illuminated focal point in a $3 \mathrm{D}$ space to produce a 3D structure (Hanada et al., 2011; Hwang et al., 2017). The block diagram of direct laser writing is shown in Figure 3C.

\section{Inkjet Printing}

In this $3 \mathrm{D}$ printing technique, powder particles are spread over the platform, and the hydrogel droplets or low-viscosity photocurable resin are used as printing materials. Liquid composition materials help to bind the powder particles to a solid of sufficient strength. Each layer can be constructed by ejecting the ink from the fine deposition nozzle, and the 3D model can be produced by a laser approach. Two types of inks are used in this technique for printing the object, one is wax-based and the other is liquid-based (Han et al., 2019).

\section{Bioprinting}

$3 \mathrm{D}$ bioprinting is an additive manufacturing process in which organs and tissues are printed three dimensionally using a layerby-layer method (Mukherjee et al., 2019). There are three stages in the $3 \mathrm{D}$ bioprinting process: pre-bioprinting, bioprinting, and postprinting. Bioprinting technologies, according to their working mechanism, can be divided into four main modalities: 1) inkjet-based bioprinting, 2) extrusion-based bioprinting, 3) stereolithography, and 4) laser-based bioprinting (Iordache, 2019). The ink used for bioprinting is commonly referred to as "bioink." In general, this bioink consists of living cells when printing tissues or organs, whereas in the case of printing scaffolds, it does not contain any living cells. Besides cells, the bioink consists of several polymer compositions in which the cells are suspended. Among the commonly used synthetic polymers in the bioprinting field are polycaprolactone (PCL), poly-l-lactic acid (PLLA), poly (lactic-co-glycolic acid) (PLGA), and poly (ethylene glycol) (PEG). The composite of synthetic and natural polymers offers tuneable viscosity, with high functionality (Papaioannou et al., 2019). Different 3D printing techniques were compared in Table 1 on the basis of their working principle, material used, advantages, and disadvantages, for different biomedical application.

\section{BIOMEDICAL APPLICATION FOR 3D PRINTING MICROFLUIDICS}

In this section, we are discussing different 3D-printed microfluidic-based miniaturized devices and their implications toward different biomedical applications. A special focus has been emphasized on compiling the studies related to the designing of 3D-printed microfluidic-based sensors for various biomedical applications. On the road to this, Lee et al. have created hydrogel scaffolds containing fluidic channels to generate perfusable $3 \mathrm{D}$ artificial tissue composites by $3 \mathrm{D}$ bioprinting technique (Lee et al., 2010). The goal of this work is to simultaneously handle and print phase-transition materials, such as pH-sensitive, thermosensitive, photocurable, or chemically cross-linkable hydrogels, along with various cell types, which are essential for the creation of complex tissue- like hydrogel scaffolds. The collagen hydrogel precursor is printed and cross-linked through a nebulized sodium bicarbonate solution. The heated gelatin solution that acts as a sacrificial factor for the fluidic channels was printed between the layers of collagen. To achieve 3D hydrogel block, the process was repeated layer by layer. This printed hydrogel block was then heated at $37^{\circ} \mathrm{C}$, which allowed gelatin to liquefy selectively and drain, generating a hallow channel within the collagen scaffold. Interestingly, the human dermal fibroblast cells grown in the scaffolds with microfluidic channels showed better proliferation than the scaffold without the channels. Thus, integrating the on-demand capability of the $3 \mathrm{D}$ printing technique to print fluidic channel structures and cells in 3D scaffold architecture offers prospects to generate perfusable $3 \mathrm{D}$ artificial tissue composites. Yeong et al. fabricated porous PCL scaffolds for cardiac tissue engineering by selective laser sintering $3 \mathrm{D}$ printing technique (Yeong et al., 2010). In this method, a cardiac cell seeded construct is cultured in vitro before transplantation to the injured heart via minimally invasive surgery. C2C12 myoblasts have been cultivated on the scaffold to investigate the cellularity of the scaffold design for up to 21 days. The cell culture studies reveal that the scaffolds got densely populated with the growing cells even at 4 days postseeding (p.s.) and starts fusion and differentiation as early as 6 days p.s., which was confirmed using myosin heavy-chain immunostaining on 11th day p.s. Interesting, a steady number of cells were then maintained throughout 21 days of culturing.

$\mathrm{Wu}$ et al. fabricated 3D biomimetic microvascular networks embedded in the hydrogel matrix by omnidirectional printing (Wu et al., 2011). In this method, fugitive ink filaments are printed inside a photocurable gel reservoir that physically supports the patterned features, thereby enabling completely omnidirectional freeform fabrication. These hydrogel-based microvascular structures may have potential applications in $3 \mathrm{D}$ cell culture, tissue engineering, organ modeling, or autonomic healing. Wang et al. fabricated the 3D microfluidic origami device integrated with electrochemiluminescence (ECL) immunosensor for point-ofcare detection of carcinoma antigen 125 (Figure 4A) (Wang et al., 2013). This ECL-based immunosensor device was manufactured by direct screen printing the carbon working counter electrodes and $\mathrm{Ag} / \mathrm{AgCl}$ reference electrodes with their conductive pads on wax-patterned pure cellulose paper and was activated by folding to form a 3D electrochemical cell. To achieve high sensitivity, the working electrode was modified with gold nanoparticles (AuNPs) immobilized with first capture antibodies (Figure 4B). This microfluidic origami ECL immunodevice revealed a good linearity range from 0.01 to $100 \mathrm{U} \mathrm{mL}^{-1}$ and a detection limit of $0.0074 \mathrm{U} \mathrm{mL}^{-1}$ with good stability and sensitivity. The authors have proposed the possible application of this immunodevice in point-of-care testing of different tumor markers for remote regions and developing countries.

Toward the development of a rapid, sensitive, and specific detection system for influenza virus, Krejcova et al. have fabricated 3D-printed bead-based microfluidic chip (Krejcova et al., 2014). The working of the microfluidic device involves a 


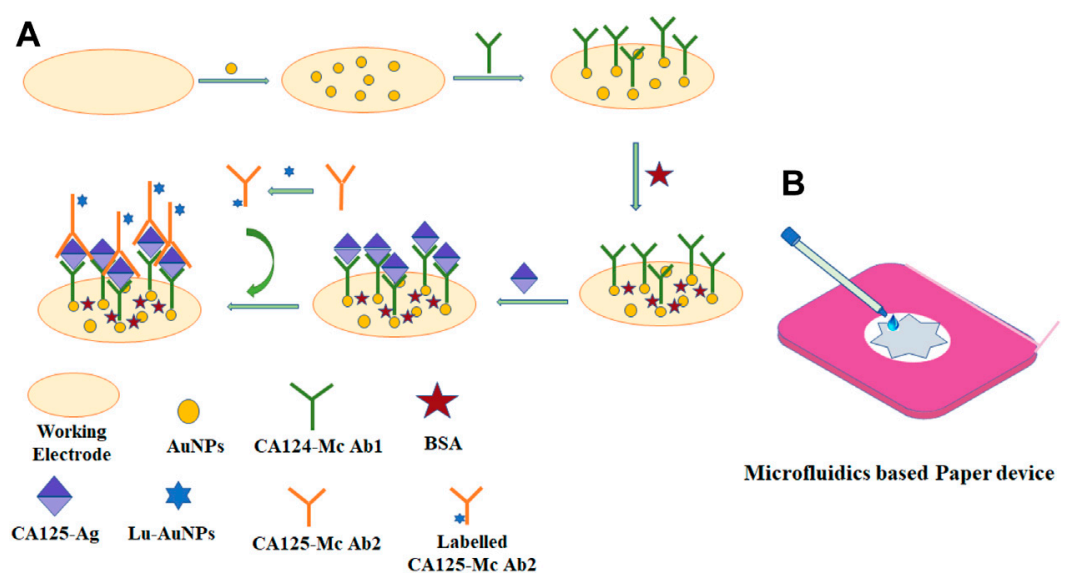

FIGURE 4 | Schematic representation of (A) fabrication of the ECL immunosensor and assay procedure and (B) 3D microfluidic origami immunodevice with transparent device-holder.

two-step procedure including the isolation involving the paramagnetic beads (MPs) and electrochemical detection. For the isolation process, the platform was created with streptavidinmodified MPs, which were conjugated via biotinylated glycan, followed by linkage of hemagglutinin to the glycan. Vaccine hemagglutinin (HAvaxi) was labeled with CdS quantum dots (HA-QDs) at first for detection purposes. Detection of the isolation product by voltammetry was the end point of the procedure. To design the $3 \mathrm{D}$-printed chips, the FDM technique using polylactide material was used. This 3Dprinted microfluidic system is emphasized to be a very promising and powerful tool for rapid influenza antigen diagnosis, and this approach can also be applied for the diagnosis of other pathogens. Lee et al. fabricated a novel method for the rapid detection of pathogenic bacteria using an immunomagnetic assay based on a 3D-printed microfluidic device and a luminescent ATP detection kit (Lee et al., 2014). In this work, binding between the antibody-functionalized magnetic nanoparticle clusters (AbMNCs) and the bacterium Salmonella was facilitated by magnetic immobilization of the AbMNCs on the surface of a three-dimensional microchannel in a hollow cylinder. This high capacity and sensitive diagnostic system showed a lower detection limit of $10 \mathrm{cfu} / \mathrm{ml}$ with a response time of $10 \mathrm{~min}$. Erkal et al. fabricated two 3Dprinted devices with acrylate-based polymer material using Objet Connex 350 multi-material printers for electrochemical detection of NO and dopamine (Figure 5) (Erkal et al., 2014). In both the printed devices, the electrode is housed in commercially available polymer-based fittings so that the various electrode materials (platinum, platinum black, carbon, gold, and silver) can be easily added to a threaded receiving port printed on the device. This will provide a modulelike style to the experimental design, where the electrodes can be removed, repolished, and reused after biological sample treatment. The first printed device embodies a microfluidic stage with a $500 \times 500-\mu \mathrm{m}$ channel and a threaded receiving port to permit the integration of either polyetheretherketone
(PEEK) nut-encased platinum black or glassy carbon electrodes for nitric oxide (NO) and dopamine detection, respectively. The embedded $\mathrm{Pt} / \mathrm{Pt}$-black electrode was reported to have a limit of detection of $1 \mu \mathrm{M}$ with a broad linearity range of 7.6-190 $\mu \mathrm{M}$ for NO gas, whereas with the glassy carbon electrode, the device showed the detection range of $25-500 \mu \mathrm{M}$ with LOD of $500 \mathrm{nM}$ for dopamine. The second 3D-printed fluidic device is reported to allow the assortment of biologically relevant analyte ATP and measures its release signal simultaneously.

Chen et al. have developed 3D-printed microfluidic device using Objet Connex 350 printers and successfully used it to facilitate the quantitative determination of ATP release from erythrocytes (ERYs) stored under different conditions by the use of a plate reader (Chen et al., 2014). They stored ERYs in AS-1 (a currently approved storage solution) and AS-1N (AS-1 with modified glucose concentrations) were circulated and checked on a single $3 \mathrm{D}$ printing unit, greatly improving the effectiveness of the experimental data by reducing the uncertainty of using different devices. Six channels incorporated into the system allow high-throughput flow analysis, and the static wells between channels facilitate simultaneous internal standards and/or calibrators. Roda et al. developed a smartphone-based chemiluminescence biosensor for noninvasive and easy monitoring of the endurance performance of athletes through lactate detection (Roda et al., 2014). Disposable mini cartridge fabricated by the FDM 3 D printing technology can easily be prototyped to turn any kind of smartphone or tablet into portable luminometer to detect chemiluminescence derived from enzymecoupled reactions. As a proof of concept, lactate oxidase was combined with horseradish peroxidase for the determination of lactate in oral fluid and sweat. Lactate can be measured in less than 5 mins with detection limits of 0.5 and $0.1 \mathrm{mmol} / \mathrm{L}$ in oral fluid and sweat. Chudobova et al. fabricated a 3D-printed chip, with gold nanoparticle (AuNP) probes as methicillin-resistant Staphylococcus aureus (MRSA) indicators, which are suitable for bacterial cultivation, DNA isolation, PCR, and detection of amplified genes (Chudobova et al., 2014). The detection of 


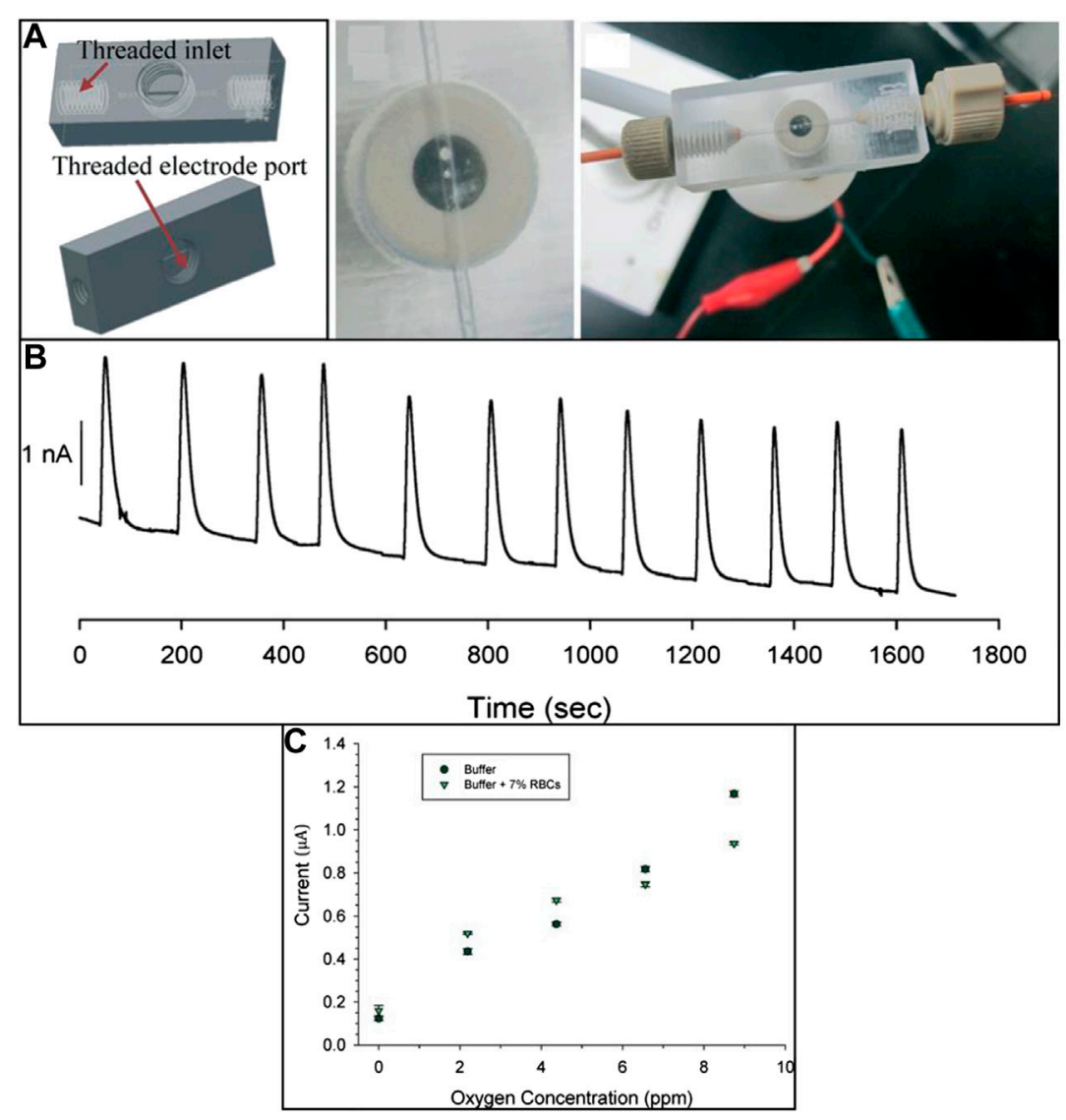

FIGURE 5 | (A) 3D-printed device with 0.5-mm wide microfluidic channels designed for the electrochemical detection of dopamine, nitrous oxide, and oxygen concentration. (B) Amperogram of reproducible $190 \mu \mathrm{M}$ NO injections over the Pt/Pt-black electrode. (C) Calibration curve data for oxygen standards in the buffer and the presence of RBC (reproduced with permission from Erkal et al., 2014).

MRSA in the samples was based on the specific interactions between the mecA gene with AuNP probes and the colorimetric detection, which used the non-cross-linking aggregation phenomenon of DNA-functionalized AuNPs. This system was capable of colorimetric determination of the MRSA in a microfluidic reactor, allowing it to be used for in vitro diagnostics.

Wearable sensors connected to sensor networks of the body can deliver real-time information about the wearer's health and fitness, which are of great interest commercially. In this lane, Gowers et al. for the first time developed a 3D-printed wearable microfluidic device integrated with FDA-approved clinical microdialysis probes and needle-type biosensors for constant monitoring of the human tissue metabolite levels (Gowers et al., 2015) (Figure 6). The authors demonstrated the potential of this $3 \mathrm{D}$-printed microfluidic system as a wearable device for subcutaneous monitoring of tissue glucose and lactate levels in cyclists during exercise. The clear changes recorded in the local glucose and lactate levels indicate that this device could have huge potential for real-time monitoring and assessing athlete training effectiveness.

Bishop et al. used the stereolithography technique for fabricating the $3 \mathrm{D}$-printed microfluidic device which is integrated with a biosensor electrode for the measurement of electrochemiluminescence signal (Figure 7) (Bishop et al., 2015). The ECL generated from $\left[\mathrm{Ru}(\mathrm{bpy})_{3}\right]^{2+}$ in TPA buffer solution or DNA on a pencil graphite electrode was readily detected using a CCD bioimaging camera. The results derived from this study indicate that this $3 \mathrm{D}$-printed platform can serve as an effective platform for designing more advanced and low-cost ECL-based sensing systems. Kadimisetty et al. have developed a novel, low-cost, gravity-flow microfluidic immunosensor with polylactide acid (PLA) using fused deposition modeling technique for detection of three cancer biomarker proteins (Kadimisetty et al., 2016). This supercapacitor-powered electrochemiluminescence (ECL) protein immune array detects simultaneously prostate-specific antigens (PSAs), prostatespecific membrane antigens (PSMAs), platelet factor-4 (PF-4), and prostate cancer biomarkers within $35 \mathrm{~min}$. This technology will provide responsive on-site cancer diagnostic in resourcelimited environments and require only minor training.

Ragone et al. fabricated a portable and disposable electrochemical sensor for the "downward" sensing of metabolites or excreted biologically active molecules (Ragones et al., 2015). The rapid detection capability of biomarker alkaline phosphatase (ALP) excreted from colon cancer cell lines was demonstrated. The microfluid chip 


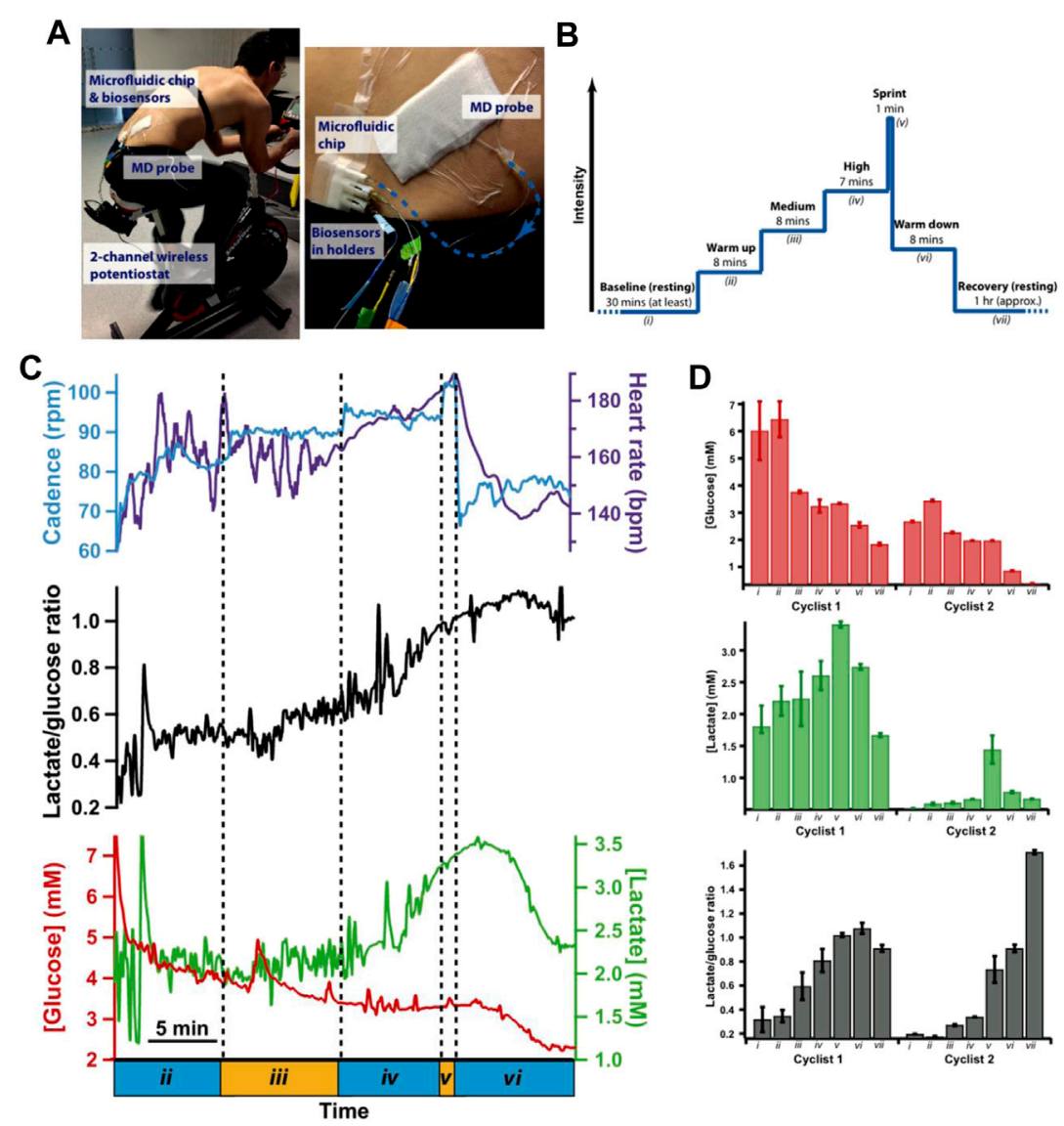

FIGURE 6 | (A) Photograph of a microfluidic device to measure tissue glucose and lactate levels in dialysate during the cycling protocol, (B) experimental protocol, (C) dialysate glucose, and lactate levels during the exercise phase of the cycling protocol system. (D) Histograms showing mean dialysate levels for two different cyclists during key points in the cycling protocol (reproduced with permission from Gowers et al., 2015).
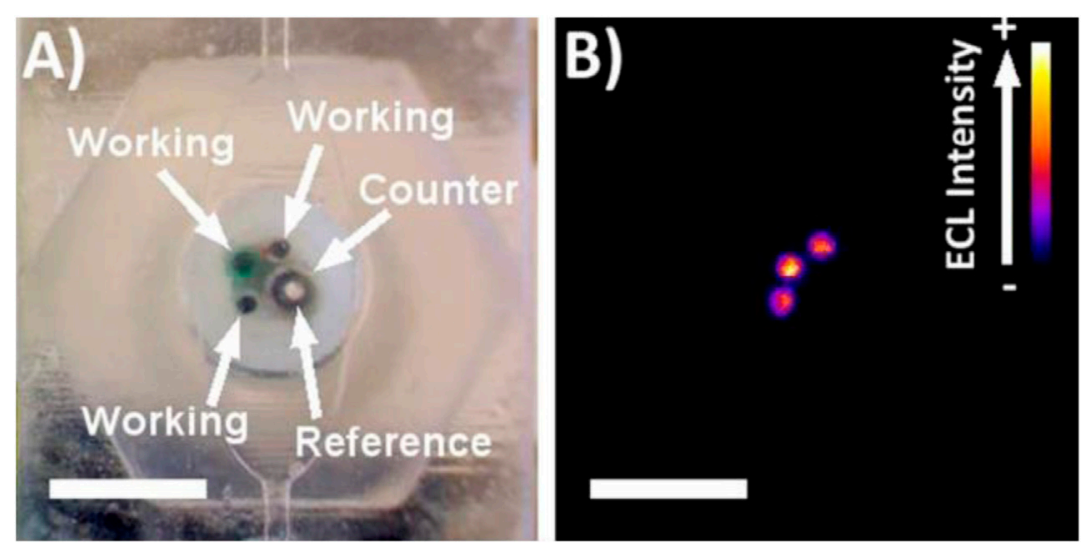

FIGURE 7 | (A) Picture of electrode arrays incorporated into 3D-printed channel. (B) ECL response from electrode array in $180 \mu \mathrm{M}\left[\mathrm{Ru}(\mathrm{bpy})_{3}\right]^{2+}$ in $0.2 \mathrm{M}$ phosphate buffer with $100 \mathrm{mM}$ TPA (reproduced with permission from Bishop et al., 2015).

consists of a biocompatible substrate composed of an electrochemical cell with two gold electrodes as working and counter electrodes and an $\mathrm{Ag} / \mathrm{AgCl}$ electrode as a quasi- reference electrode and it was fabricated by stereolithography 3D printing technique. The electroactivity of working electrodes was verified by cyclic voltammetry of a ferrocyanide/ 


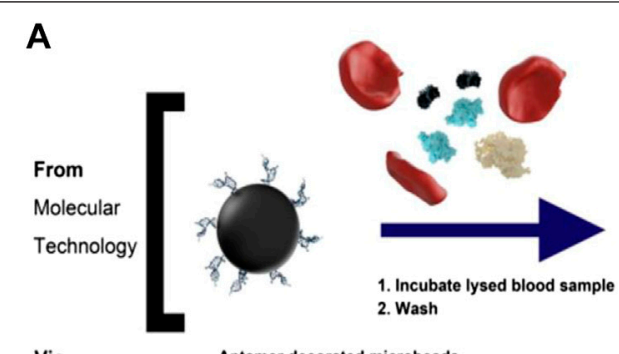

Via Aptamer decorated microbeads
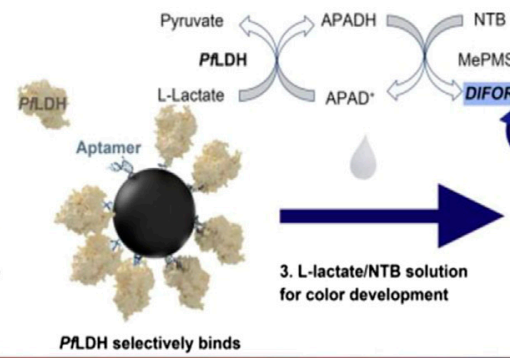

NTB IFORMAZAN 3D Prototyping

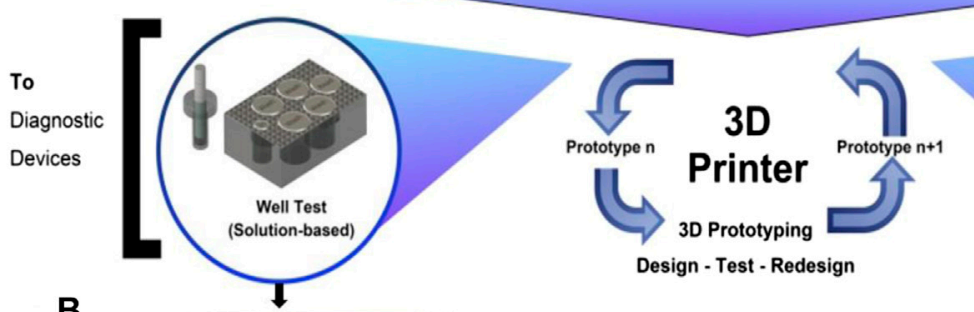

B

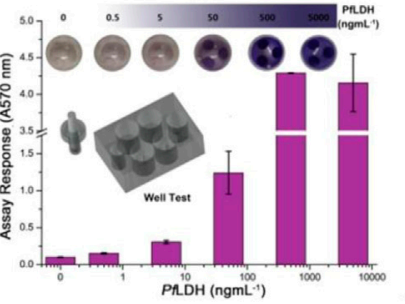

C

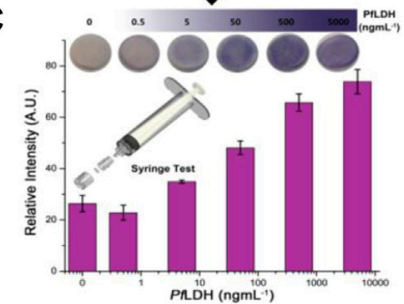

FIGURE 8 | (A) 96-well malaria-based assay (APTEC) was modified to microbead based assay for improved functionality. The assay functions using aptamerfunctionalized microbeads to collect PfLDH from patient blood samples. The production reagent is applied after washing, which creates a blue response for malariapositive samples, using the enzymatic activity of PfLDH. (B,C) The response of prototypes of APTEC point-of-care devices using different PfLDH concentrations in serum samples (5\% BSA) for the syringe test and well test which turns blue when positive. The response was quantified using absorbance (A570 nm) (reproduce with permission from Dirkzwager et al., 2016).

ferricyanide redox reaction. Amperometric in vitro detection of the biomarker alkaline phosphate was successfully demonstrated directly in a cell culture plate from three separate colon cancer lines while preserving their biological environment. Cevenini et al. have reported a compact standalone toxicity sensor that integrates bioluminescent cells into a smartphone-based system (Cevenini et al., 2016). They fabricated 3D-printed cartridges to incorporate a variety of bioluminescent cells into ready-to-use cartridges and demonstrated the feasibility of accurate detection and quantification of BL signals. Human embryonic kidney cells (Hek293T) have been constitutively used to express greenemitting luciferase as sentinel cells, and an Android app was created to provide a user-friendly environment. The smartphone adaptor and mini cartridges were fabricated by the $3 \mathrm{D}$ printing technique.

Dirkzwager et al. aimed to develop a point-of-care malaria diagnostic test using the adaption of an aptamer-tethered enzyme capture (APTEC) sensing system using 3D printing technology as a platform for rapid prototyping (Dirkzwager et al., 2016). The assay functions by capturing the malaria biomarker Plasmodium falciparum lactate dehydrogenase $(\mathrm{PfLDH})$ from samples and using its intrinsic enzymatic activity to generate a visualizable blue color in response to Plasmodium-positive samples (Figure 8). With the help of the stereolithography 3D printing technique, they developed two prototypes, magnetic bead-based well test and paper-based syringe test. Both were found to have been effective in detecting recombinant $\mathrm{PfLDH}$ at $\mathrm{ngmL}^{-1}$ concentration using low sample volumes $(20 \mu \mathrm{L})$ and could work using distilled or spiked whole blood samples with easy sample preparation. The syringe test required additional preparation for measuring and found to be more analytically sensitive, while the magnetic bead-based well test required fewer steps and could therefore be ideally suitable for future clinical trials.

$\mathrm{Su}$ et al. fabricated flow bioreactors using a commercial $3 \mathrm{D}$ FDM-type printer and developed a simple procedure for the functionalization of printed ABS reactors to facilitate the determination of glucose and lactate in biological samples (Su et al., 2016). For monitoring the concentration of the rat brain extracellular glucose and lactate (Figure 9), this system involved microdialysis (MD) sampling and fluorescence determination in conjunction with a novel sample derivatization scheme in which glucose oxidase and lactate oxidase were immobilized in ABS flow bioreactors. To demonstrate the system's applicability, 1) spike analysis of 


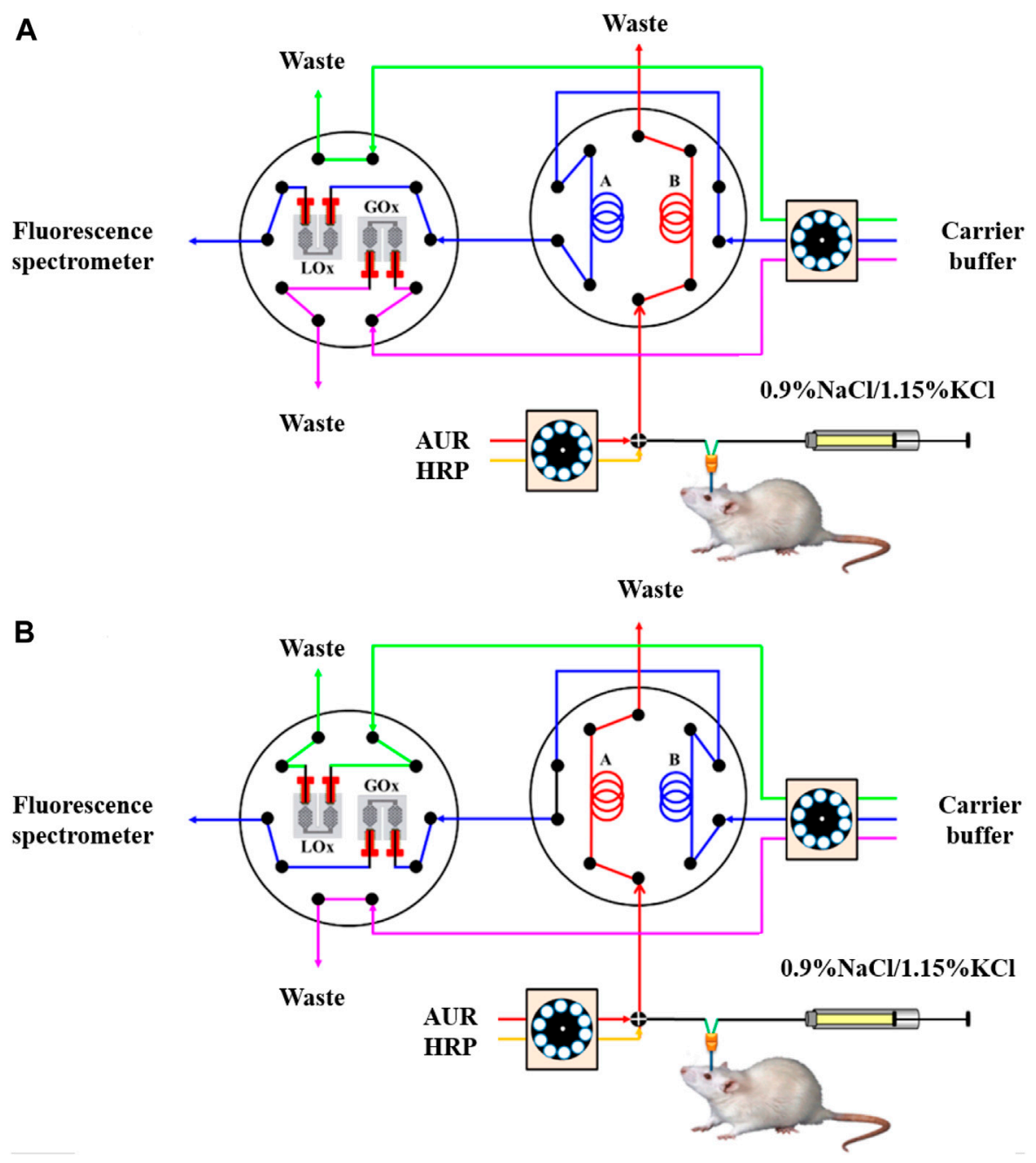

FIGURE 9|Schematic representation of the proposed online glucose/lactate monitoring system. (A) Determination of lactate: The microdialysate was mixed online with two streams of AUR and HRP solutions and then loaded into sample loop A, while the conditioned microdialysate in sample loop B was transferred via the LOximmobilized bioreactor to the fluorescence spectrometer. (B) Determination of glucose: The microdialysate was conditioned online and then loaded into sample loop B, while the conditioned microdialysate in sample loop A was transferred via the GOx-immobilized bioreactor to the fluorescence spectrometer (Reproduced with permission from Su et al., 2016).

offline-collected rat brain microdialysate and 2) in vivo dynamic monitoring of extracellular glucose and lactate in live rat brains, in addition to inducing neuronal depolarization by perfusing a high- $\mathrm{K}^{+}$medium from the implanted MD probe. Tang et al. developed the $3 \mathrm{D}$-printed microfluidic system for automatic detection of cancer biomarker proteins using the stereolithography technique (Tang et al., 2017). This unibody consists of 1) three reagent reservoirs, 2) an effective 3D network for passive mixing, and 3) an optically transparent detection chamber housing in a glass slide decorated capture antibodies array for observing chemiluminescence output with CCD camera (Figures 10A,B). This low-cost automatic system was used for the diagnosis of cancer biomarker proteins such as platelet factor 4 (PF-4) and prostate-specific antigen (PSA). The detection limit of the sensor device is estimated to be $0.5 \mathrm{pg} \mathrm{ml}^{-1}$, and good accuracy vs. ELISA was authenticated by analyzing human serum samples. This research system holds great promise for further advancement as a diagnostic device for early cancer treatment.

Sibbitt et al. fabricated the 3D microfluidic device by stereolithography technique (Sibbitt and He, 2017). The robustness and usability of these protocols allow the incorporation of modular $3 \mathrm{D}$ design and microfabrication of POC microfluidics integrated with smartphone-based interfaces as stand-alone disease diagnostic devices. It enables rapid exploration of biological phenomena at the microfluidic level by making microfluidic technology readily and easily accessible to researchers unfamiliar with microfabrication, expanding the reach of detection capabilities in POC microfluidic devices. Park et al. 

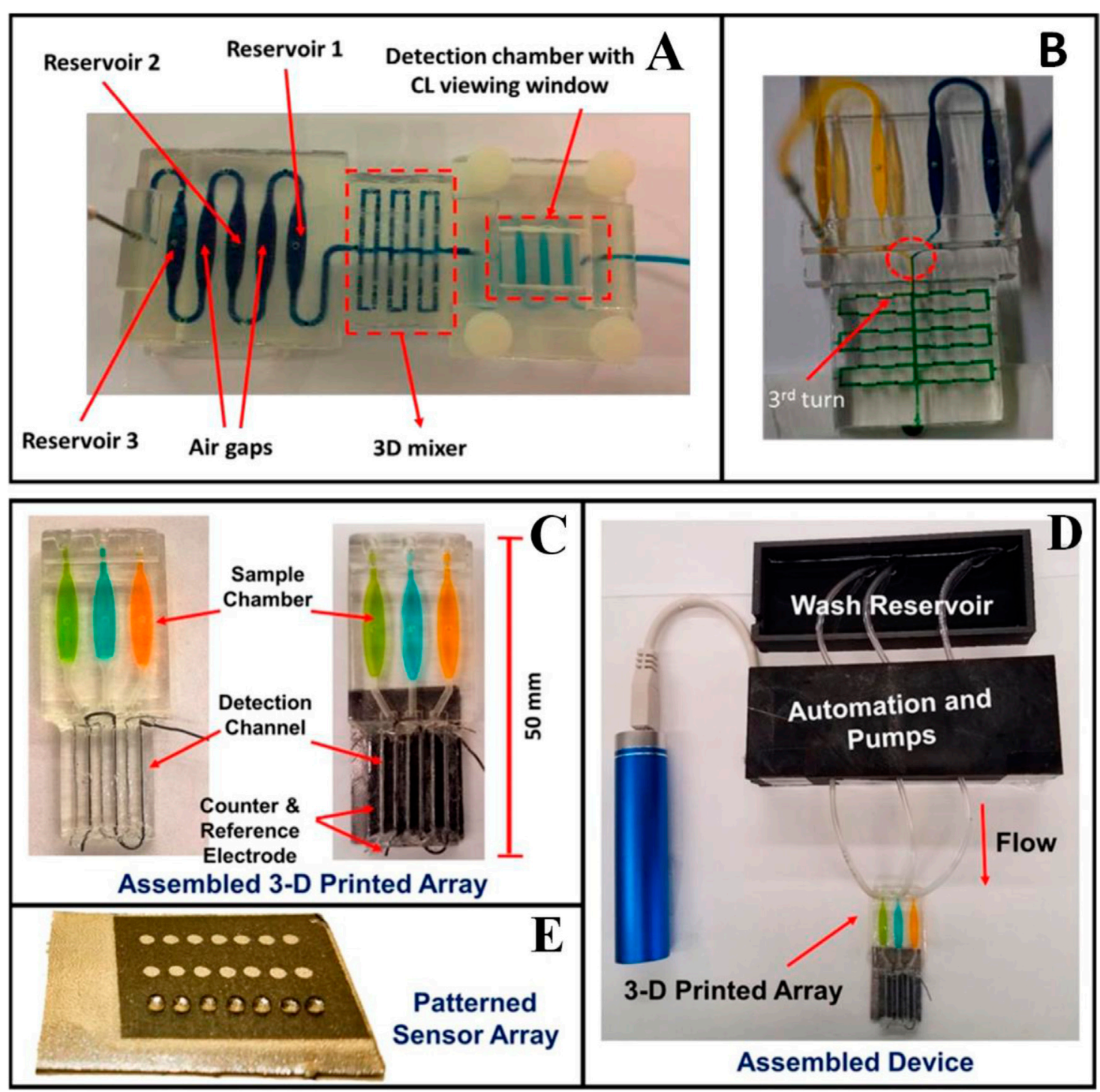

FIGURE 10 | (A) 3D-printed unibody CL immunoarray device. (B) A modified 3D-printed device to demonstrate the mixing ability of the passive 3D mixing network. (C) 3D-printed Automated genotoxicity screening device without or with microwell chip and counter electrode wires inserted showing the sample chamber dye solutions. (D) Microwell-patterned pyrolytic graphite detection array showing the first-row holding $1 \mu$ l water droplets retained by the hydrophobic microwell boundaries. Each row is fed by a separate sample line. The working array features films of DNA, metabolic enzymes, and RuPVP in each microwell. (E) Assembled array system showing box enclosing electronic microprocessors and micropumps driven by a rechargeable battery and connected to the 3-D printed array below with a wash reservoir with a containing pH 7.4 buffer. (Reproduced with permission from Tang et al., 2017 and Kadimisetty et al., 2017).

fabricated a 3D microfluidic magnetic preconcentrator $(3 \mathrm{D} \mu \mathrm{FMP})$ which was used for the recognition of bacterial pathogen (Escherichia coli 0157:H7) (Park et al., 2017). E. coli 0157:H7 induces hemorrhagic colitis (HC) and hemorrhagic uremic syndrome (HUS) in a person who eats food infected with this microorganism. A 3D microfluidic magnetic preconcentrator is fabricated by a digital light processing $3 \mathrm{D}$ printer which is capable to selectively preconcentrate this bacterial pathogen in large-volume sample solutions into small volumes at the submillimeter scale by using specific antibody-conjugated magnetic nanoparticles for the pathogen. The optical determination was accomplished through the use of a commercial ATP luminometer, where the ATP present on the preconcentrated bacterial cells was measured and quantified by the light emitted during each measurement, yielding a detection limit as low as $10 \mathrm{cfu} / \mathrm{ml}$ in blood. Kadimisetty et al. fabricated 3D-printed microfluidic array for the detection of the genotoxic potential of cigarettes, e-cigarette, and environmental samples (Figures 10C-E) (Kadimisetty et al., 2017). The microfluidic array is developed by using the stereolithography technique. Electronic cigarettes are battery-powered devices that vaporize nicotine and have been designed as an alternative to a tobacco cigarette; it is a very harmful substance for 

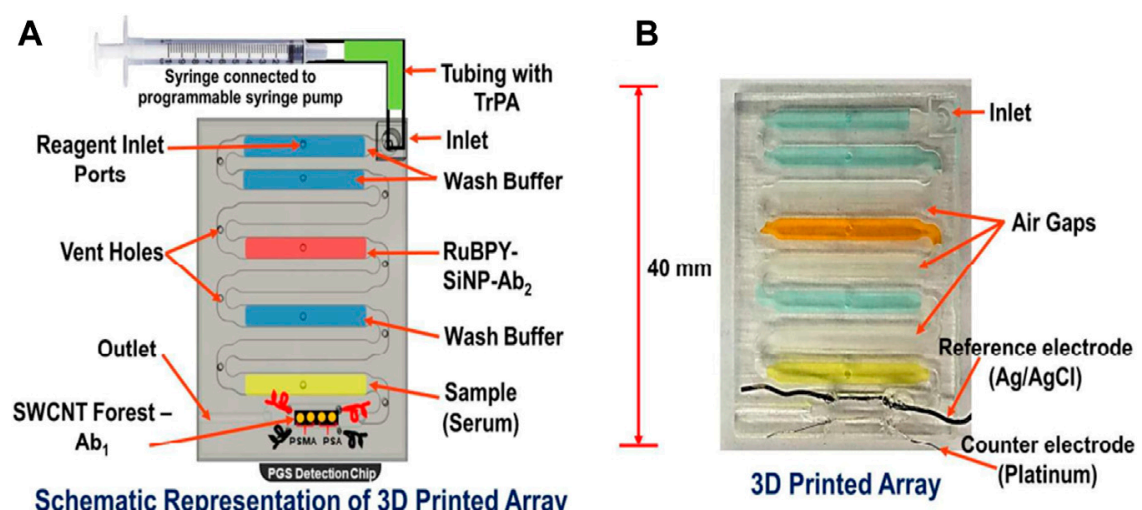

FIGURE 11 | (A) Model of an array with labelled reagent chambers connected to 4 microwells pyrolytic graphite (PGS) detection chip. (B) Picture of a 3D printed microfluidic array and fluidic chambers filled with colored dyes for identification. (Reproduced with permission from Kadimisetty et al., 2018b).

humans. The electrochemical luminescent (ECL) detection platform combines layer-by-layer film-assembled microsomal enzymes, DNA, and ECL-emitting ruthenium metallopolymers at a depth of $10 \mathrm{~nm}$.

Santangelo et al. fabricated 3D printed microfluidic chip by using the stereolithography $3 \mathrm{D}$ printing technique which is coupled with the silicon photomultiplier (SiPM) (Santangelo et al., 2017). This 3D printed microfluidic chip is used for testing a miniaturized bioluminescence sensing system for highly sensitive real-time ATP detection. This interface allowed the sample solution to be delivered close to the SiPM to improve the detection efficiency of the bioluminescence light emitted from the sample solution. The use of SiPM as a detector greatly simplifies the analysis of signals compared to conventional systems, offering quantitative performance signals that do not require post-production of images and/or off-line analysis. Fraser et al. used stereolithography to fabricate the microfluidic device for malaria diagnosis (Fraser et al., 2018). They proposed a new design for malaria biosensors in which magnetic microbeads are coated with aptamers for magnet-guided capture, wash, and detection of the biomarker. A biosensor incorporating three separate microfluidic chambers was designed to enable such magnet guided equipment-free colorimetric detection of PfLDH. The biosensor showed high sensitivity and specificity when detecting PfLDH using both in vitro cultured parasite samples and using clinical samples from malaria patients. Kadimisetty et al. have demonstrated the use of stereolithography $3 \mathrm{D}$ printing technique to fabricate the microfluidic molecular diagnostic device involving nucleic acid amplification for preventing and treating infectious diseases (Kadimisetty et al., 2018a). They tested different surface coatings to enhance the biocompatibility of $3 \mathrm{D}$ printed microfluidic device, and showed that PEG coating provided the best results for nucleic acid-base molecular diagnostic. This device enables quick molecular diagnostic testing for infectious diseases at the point of diagnosis. They also demonstrated the suitability of the device for both real-time quantitative detection of fluorescence and end point colorimetric qualitative detection. This diagnostic device was used to detect Neisseria meningitides in cerebrospinal fluid (CSF) samples and Plasmodium falciparum in plasma samples by loop-mediated, isothermal amplification (LAMP) in less time. This simple and affordable 3D printing tool has tremendous potential for POC molecular disease diagnosis in resource-constrained settings. In another report, Kadimisetty et al. demonstrated a $3 \mathrm{D}$ printed electrochemiluminescence (ECL) immunoarray for simultaneous detection of two proteins (Prostate-specific antigen and Prostate-specific membrane antigen) at the same time (Figures 11A,B) (Kadimisetty et al., 2018b). This 3D printed chip is combined with a nano pyrolytic graphite sheet microwell chip for detection of the same. Stereolithographic techniques are used to print this $3 \mathrm{D}$ device. With this program, a programmable syringe pump is used and allows the pump to halt and restore the reagent delivery as necessary for the completion of the sandwich immunoarrays. The designed device is reported to be very cost-effective with low detection time using a small sample size.

Diabetes has become a global public health epidemic. For testing the insulin level, Yao et al. have developed the pneumatic microfluidic device using a 3D-based extrusion printer for insulin detection using impedance spectroscopy measurement (Yao et al., 2018). The present research offers an important technological approach to the production of low-cost microfluidic tools for efficient reagent mixing in microscale biochemical detection systems. Oh et al. developed and fabricated a 3D-printed blood viscosity analysis (Oh and Choi, 2018). Viscosity measurement is essential for the quality assurance of liquid products, as well as for monitoring the viscosity of clinical fluids as a potential hemodynamic biomarker. They demonstrated multiplexed viscosity measurements of Newtonian fluids of varying viscosities and viscosity measurements of non-Newtonian fluid at different shear rate conditions. Samper et al. have developed and manufactured 3D-printed microfluidic device which can be easily integrated with the electrochemical biosensor for the detection of biomarkers such as glucose, lactate, and glutamate (Samper et al., 2019). The time resolution of the device is characterized by recording short lactate concentration pulses. 
TABLE 1 | Comparative analysis of various 3D printing methods with a special focus on materials used, their benefits, and drawbacks.

\begin{tabular}{|c|c|c|c|c|}
\hline Technique & Principle & Material & Advantage & Disadvantage \\
\hline SLA & $\begin{array}{l}\text { UV initiated polymerization } \\
\text { cross section by cross section }\end{array}$ & $\begin{array}{l}\text { Resin (epoxy based with } \\
\text { proprietary or acrylate) }\end{array}$ & $\begin{array}{l}\text { Large part can be easily manufactured, } \\
\text { uncured material can be used, high } \\
\text { accuracy, good for complex built, and good } \\
\text { scalability }\end{array}$ & $\begin{array}{l}\text { Slow building process, expensive, } \\
\text { reduction in the durability of the product due } \\
\text { to the involvement of moisture, heat, and } \\
\text { chemicals }\end{array}$ \\
\hline DLP & $\begin{array}{l}\text { Photocuring by a digital } \\
\text { projector screen to project } \\
\text { layers by squared voxels. }\end{array}$ & $\begin{array}{l}\text { Photo-resin and } \\
\text { photopolymer }\end{array}$ & $\begin{array}{l}\text { Excellent accuracy of laying, high resolution, } \\
\text { and uncured photopolymer can be reused }\end{array}$ & $\begin{array}{l}\text { Insecurity of the consumable material and } \\
\text { difficult to print large structure }\end{array}$ \\
\hline SLS & $\begin{array}{l}\text { Laser induced sintering of } \\
\text { powder particles }\end{array}$ & $\begin{array}{l}\text { PVC, metallic powder, and } \\
\text { polyamide }\end{array}$ & High strength and high resolution & $\begin{array}{l}\text { Only metal parts can print, finishing or } \\
\text { postprocessing required due to its grainy } \\
\text { roughness, and difficulty in material change } \\
\text { over }\end{array}$ \\
\hline $\begin{array}{l}\text { PolyJet } \\
\text { process }\end{array}$ & $\begin{array}{l}\text { Deposition of the droplets of the } \\
\text { photocurable liquid material } \\
\text { and cured }\end{array}$ & Polymer & $\begin{array}{l}\text { Multiple jetting heads are available to build } \\
\text { materials, different levels of flexibility, allows } \\
\text { using different colored photopolymers, high } \\
\text { accuracy, and smooth surface }\end{array}$ & $\begin{array}{l}\text { Vulnerable to heat and humidity, lose } \\
\text { strength over time, relatively higher cost } \\
\text { than others, and sharp edges are often } \\
\text { slightly rounded. }\end{array}$ \\
\hline LOM & Paper cutting & $\begin{array}{l}\text { Sheet metals, paper, } \\
\text { cellulose, and plastic }\end{array}$ & $\begin{array}{l}\text { Large parts can easily manufacture and low } \\
\text { cost }\end{array}$ & $\begin{array}{l}\text { Time-consuming and specific material } \\
\text { types can be used }\end{array}$ \\
\hline DLW & Laser-based & Fused silica, glass, etc. & Colorful printing & $\begin{array}{l}\text { Post-surface treatment is required and low } \\
\text { strength }\end{array}$ \\
\hline $\begin{array}{l}\text { Inkjet } \\
\text { printing }\end{array}$ & $\begin{array}{l}\text { Extrusion of ink and powder } \\
\text { liquid binding }\end{array}$ & Hydrogel or photo-resin & $\begin{array}{l}\text { High accuracy and very high surface } \\
\text { finishes }\end{array}$ & $\begin{array}{l}\text { Slow build process, low mechanical } \\
\text { properties, to remove the moisture post } \\
\text { processing is required, the grainy or rough } \\
\text { appearance }\end{array}$ \\
\hline FDM & Extrusion-based & $\begin{array}{l}\text { Nylon, polylactic acid, wax } \\
\text { blend, acrylonitrile } \\
\text { butadiene, and styrene }\end{array}$ & $\begin{array}{l}\text { Consume less time, high quality, and high } \\
\text { speed }\end{array}$ & Weak mechanical properties \\
\hline Bioprinting & Laser/UV & PCL, PLLA, and PEG & Cheap and high speed & Lack of accuracy in positioning of droplets \\
\hline
\end{tabular}

TABLE 2 | Summary of 3D printing technique used in biomedical application.

\section{D printing}

technique

Stereolithography

Clear resin, pyrolytic graphite sheets, acrylate based resin, clear methacrylatebased resin, acrylate- and epoxy-based mixture, and PDMS

DLP

Extrusion-based technique

Inkjet 3D printing

PolyJet process

Ultra 3SP technique Acrylonitrile butadiene styrene (ABS) white

Wax printing

technique

MultiJet

Bioprinting
Casting wax material

Acrylate-based polymer

Photo-curable resin acrylate-based Polylactide and acrylonitrile butadiene styrene

Cellulose paper and filter paper

Visijet M3 crystal polymer82 and Visijets 100 hydroxylated wax83

Photocurable resin, hydrogels, and viscous Making of vascular channel materials

Detection Escherichia coli toxicity biosensor

Cancer detection glucose, and lactate chemosensitivity
Applications

Detection of Salmonella bacteria, detection of electrochemiluminescence, sensing of metabolites detection of cancer biomarker proteins, diagnosis of malaria detection of genotoxic potential, diagnosis the malaria, detection of ATP, detection of prostatespecific antigen, monitoring the blood viscosity, and detection of Salmonella typhimurium DNA by LAMP Influenza virus, detection of lactate detection of cancer biomarkers, detection of glucose and lactate, detection of insulin, detection of MRSA, andsmartphone-based

Oxygen recognition in the streamlining of red blood cells, nitric oxide detection and neurotransmitter detection, and detection of ATP

Detection the level of glucose and lactate, glutamate,

Carcinoma antigen 125, cell viability, and

Treatment of an AMR infection
References

Lee et al. (2014); Bishop et al. (2015); Ragones et al. (2015); Tang et al. (2017); Dirkzwager et al. (2016); Kadimisetty et al. (2017); Sibbitt and He, (2017); Santangelo et al. (2017); Fraser et al. (2018);

Kadimisetty et al. (2018a); Kadimisetty et al. (2018b); Oh and Choi (2018); Ruiz et al. (2020)

Park et al. (2017)

Krejcova et al. (2014); Roda et al. (2014); Kadimisetty et al. (2016); Su et al. (2016); Yao et al. (2018); Chudobova et al. (2014); Cevenini et al. (2016); Zargaryan et al.

(2020)

Chen et al. (2019)

Erkal et al. (2014); Chen et al. (2014)

Gowers et al. (2015); Samper et al. (2019) Wang et al. (2013); Fu et al. (2020)

Sweet et al. (2020)

Lee et al. (2010); Wu et al. (2011)
The device is employed to record simultaneous glutamate, glucose, and lactate concentration changes simulating the physiological response to spreading depolarization events in the cerebrospinal fluid dialysate. The system is also used in the ICU to monitor a patient's brain injury remotely, demonstrating its potential for clinical surveillance. Chen et al. designed a 3D- 
printed microfluidic device to separate circulating tumor cells from human blood samples (Chen et al., 2019). The inner structure of microfluidic channels is functionalized with antiEpCAM antibodies to have a clear capture of successful human cell lines of EpCAM (such as colon cancer SW480, prostate cancer PC3, and breast cancer MCF-7). Sweet et al. developed a 3D $\mu$-concentration gradient generator ( $\mu$-CGG) prototype with the help of additive manufacturing to employing a unique $3 \mathrm{D}$ microchannel network (Sweet et al., 2020). 3D $\mu$-CGG is used to identify the optimal drug compositions through antimicrobial susceptibility testing for the treatment of antimicrobial-resistant (AMR) infections. The AMR infection occurs when bacteria, viruses, fungi, and parasites adapt over time and no longer react to antibiotics that make infections more difficult to treat and increase the risk of disease spread, serious illness, and death. The MultiJet 3D printing technique and materials Visijet M3 crystal polymer 82 and Visijets 100 hydroxylated wax 83 are used to fabricate the 3D $\mu$-CGG device.

Zargaryan et al. developed hybrid microfluidic devices that incorporate both $3 \mathrm{D}$-printed and paper-based elements. This method operates by $3 \mathrm{D}$ printing directly onto a standard laboratory filter paper using widely available commercial fused deposition modeling printers (Zargaryan et al., 2020). They designed finger-actuated reservoirs and reversible mechanical valves that can be intuitively controlled by untrained users to demonstrate the capabilities of this technology. The intuitive design of device operation can be particularly useful for self-governing point-of-care research that reduces the burden on laboratory or healthcare systems during periods of need, such as the COVID-19 pandemic or other global health crises. Ruiz et al. defined a two-stage 3D printing process for the development of hybrid microfluidic devices integrating both hard and soft materials by using lowcost 3D consumer printers (Ruiz et al., 2020). Printed hard components are first created by a stereolithography (SLA) printer moved to a second FDM printer where a soft printed part is connected to the FDM printing. Three different types of hybrid microfluidics devices are microfluidic quick connect component, finger-actuated pump, and microfluidic reactor chip with screw-seal sample inlet ports, which are used to host isothermal amplification and detection of Salmonella typhimurium DNA by loopmediated isothermal amplification (LAMP). $\mathrm{Fu}$ et al. presented a novel paper-like microfluidics 3D cell culture system to research cell viability and chemosensitivity in $3 \mathrm{D}$ environments using colorimetric measurement techniques (Fu et al., 2020). The cellulose filter paper was selected as a cell culture substrate with its advantages of biocompatibility,

\section{REFERENCES}

Ahangar, P., Cooke, M. E., Weber, M. H., and Rosenzweig, D. H. (2019). Current biomedical applications of $3 \mathrm{~d}$ printing and additive manufacturing. Appl. Sci. 9 (8), 1713. doi:10.3390/app9081713 inexpensive, enough source, and easy operation. The paperbased cell culture microfluidic device is fabricated by the wax printing 3D technique; it consisted of two layers: the upper layer was used for cell culture, while the lower layer was used for medium supply. According to the study of cell viability and chemosensitivity, the result indicates that the $3 \mathrm{D}$ culture microenvironment was effectively supplied by a paper unit. Based on this study, the anticancer drug successfully presented a specific cytotoxic effect on MCF-7 and HepG2 cultured using this wick-like paper-based system. Table 2 summarizes details about various $3 \mathrm{D}$ printing technique used in different biomedical application.

\section{CONCLUSION}

The key advantages of $3 \mathrm{D}$ printing are freedom of design, mass customization, and the ability to print complex structures with minimal waste. In this review, we have discussed various $3 \mathrm{D}$ printing techniques for designing versatile microfluidic systems for different biomedical applications. Here, we explore a broad variety of $3 \mathrm{D}$ printing technologies available and highlight those that have so far been applied to microfluidic devices. 3D printing is an emerging technique with a variety of diagnostic devices for the detection of various clinically important analytes including glucose, lactate, glutamate, and biomarkers related to malaria, cancer, etc. With the surveyed and compiled literature, we strongly believe that the $3 \mathrm{D}$ printing technology if integrated with different modern techniques including microfluidics may lead to many benefits and possibilities in the medical therapies and industries.

\section{AUTHOR CONTRIBUTIONS}

PP, RS, CD, and ND prepared the manuscript. RK, PS and AS provided their valuable inputs to further improve the manuscript.

\section{FUNDING}

We thank Dr. A. K. Srivastava, director, CSIR-AMPRI, Bhopal, India, for providing facilities. Raj Kumar Sen is thankful to the Council of Scientific and Industrial Research (CSIR), India, for the award of Junior Research Fellowship. We acknowledge the financial support received from CSIR, India (MLP 0204 and MLP 0205). We also acknowledge the financial support received from the SERB, India (IPA/2020/000130).

Amin, A., Knowlton, S., Hart, A., Yenilmez, B., Ghaderinezhad, F., Katebifar, S., et al. (2016). 3D-printed microfluidic devices. Biofabrication. 8, 022001. doi:10. 1088/1758-5090/8/2/022001

Bishop, G. W., Satterwhite-Warden, J. E., Bist, I., Chen, E., and Rusling, J. F. (2015). Electrochemiluminescence at bare and dna-coated graphite electrodes in 3dprinted fluidic devices. ACS Sens. 1 (2), 197202. doi:10.1021/acssensors.5b00156 
Bragheri, F., Martinez Vazquez, R., and Osellame, R. (2016). Three-dimensional microfabrication using two-photon polymerization. Microfluidics, 310-334. doi:10.1016/b978-0-323-35321-2.00016-9

Cevenini, L., Calabretta, M. M., Tarantino, G., Michelini, E., and Roda, A. (2016). Smartphone-interfaced 3D printed toxicity biosensor integrating bioluminescent "sentinel cells". Sens. Act. B: Chem. 225, 249-257. doi:10.1016/j.snb.2015.11.017

Chen, C., Wang, Y., Lockwood, S. Y., and Spence, D. M. (2014). 3D-printed fluidic devices enable quantitative evaluation of blood components in modified storage solutions for use in transfusion medicine. Analyst. 139 (13), 3219-3226. doi:10. 1039/c3an02357e

Chen, J., Liu, C. Y., Wang, X., Sweet, E., Liu, N., Gong, X., et al. (2019). 3D printed microfluidic devices for circulating tumor cells (CTCs) isolation. Biosens. Bioelectron. 2019, 111900. doi:10.1016/j.bios.2019.111900

Chudobova, D., Cihalova, K., Skalickova, S., Zitka, J., Rodrigo, M. A. M., Milosavljevic, V., et al. (2014). 3D-printed chip for detection of methicillin-resistant Staphylococcus aureus labeled with gold nanoparticles. Electrophoresis. 36 (3), 457466. doi:10.1002/elps. 201400321

Dirkzwager, R. M., Liang, S., and Tanner, J. A. (2016). Development of aptamerbased point-of-care diagnostic devices for malaria using three-dimensional printing rapid prototyping. ACS Sens. 1 (4), 420-426. doi:10.1021/acssensors. $5 b 00175$

Erkal, J. L., Selimovic, A., Gross, B. C., Lockwood, S. Y., Walton, E. L., McNamara, S., et al. (2014). 3D printed microfluidic devices with integrated versatile and reusable electrodes. Lab Chip. 14, 2023-2032. doi:10.1039/c4lc00171k

Fraser, L. A., Kinghorn, A. B., Dirkzwager, R. M., Liang, S., Cheung, Y. W., Lim, B., et al. (2018). A portable microfluidic aptamer-tethered enzyme capture (APTEC) biosensor for malaria diagnosis. Biosens. Bioelectron. 100, 591-596. doi:10.1016/j.bios.2017.10.001

Fu, S., Zuo, P., and Ye, B. (2020). A novel wick-like paper-based microfluidic device for 3D cell culture and anti-cancer drugs screening. Biotechnol. J. 16, 2000126. doi:10.1002/biot.202000126

Gowers, S. A. N., Curto, V. F., Seneci, C. A., Wang, C., Anastasova, S., Vadgama, P., et al. (2015). 3D printed microfluidic device with integrated biosensors for online analysis of subcutaneous human microdialysate. Anal. Chem. 87, 7763-7770. doi:10.1021/acs.analchem.5b01353

Hagedorn, Y. (2017). Laser additive manufacturing of ceramic components. Laser Addit. Manuf. 2017, 163-180. doi:10.1016/b978-0-08-100433-3.00006-3

Han, T., Kundu, S., Nag, A., and Xu, Y. (2019). 3D printed sensors for biomedical applications: a review. Sensors. 19 (7), 1706. doi:10.3390/s19071706

Hanada, Y., Sugioka, K., Shihira-Ishikawa, I., Kawano, H., Miyawaki, A., and Midorikawa, K. (2011). 3D microfluidic chips with integrated functional microelements fabricated by a femtosecond laser for studying the gliding mechanism of cyanobacteria. Lab Chip. 11 (12), 2109. doi:10.1039/c1lc20101h

Hwang, H. H., Zhu, W., Victorine, G., Lawrence, N., and Chen, S. (2017). 3DPrinting of functional biomedical microdevices via light- and extrusion-based approaches. Small Methods. 2 (2), 1700277. doi:10.1002/smtd.201700277

Iordache, F. (2019). Bioprinted scaffolds. Mater. Biomed. Eng. 2019, 35-60. doi:10. 1016/b978-0-12-816901-8.00002x

Kadimisetty, K., Malla, S., and Rusling, J. F. (2017). Automated 3-D printed arrays to evaluate genotoxic chemistry: E-cigarettes and water samples. ACS Sens. 2 (5), 670-678. doi:10.1021/acssensors.7b00118

Kadimisetty, K., Mosa, I. M., Malla, S., Wardena, J. E. S., Kuhns, T., Faria, R. C., et al. (2016). 3D-Printed supercapacitor-powered electrochemiluminescent protein immunoarray. Biosens. Bioelectron. 15, 188-193. doi:10.1016/j.bios.2015.09.017

Kadimisetty, K., Song, J., Doto, A. M., Hwang, Y., Peng, J., Mauk, M. G., et al. (2018a). Fully 3D printed integrated reactor array for point-of-care molecular diagnostics. Biosens. Bioelectron. 109, 156-163. doi:10.1016/j.bios.2018.03.009

Kadimisetty, K., Spak, A. P., Bhalerao, K. S., Sharafeldin, M., Mosa, I. M., Lee, N. H., et al. (2018b). Automated 4-sample protein immunoassays using 3D-printed microfluidics. Anal. Methods. 10 (32), 4000-4006. doi:10.1039/c8ay01271g

Knowlton, S., Hsiang Yu, C., Ersoy, F., Emadi, S., Khademhosseini, A., and Tasoglu, S. (2016). 3D-printed microfluidic chips with patterned, cell-laden hydrogel constructs. Biofabrication. 8, 025019. doi:10.1088/1758-5090/8/2/025019

Ko, D.-H., Gyak, K.-W., and Kim, D. -P. (2017). Emerging microreaction systems based on 3D printing techniques and separation technologies. J. Flow Chem. 7 (3-4), 72-81. doi:10.1556/1846.2017.00013
Krejcova, L., Nejdl, L., Rodrigo, M., Rodrigo, M. A., Zurek, M., Matousek, M., et al. (2014). 3D printed chip for electrochemical detection of influenza virus labeled with CdS quantum dots. Biosens. Bioelectron. 54, 421-427. doi:10.1016/j.bios.2013.10.031

Lee, W., Kwon, D., Chung, B., Jung, G. Y., Au, A., Folch, A., et al. (2014). Ultrarapid detection of pathogenic bacteria using a $3 \mathrm{D}$ immunomagnetic flow assay. Anal. Chem. 86 (13), 6683-6688. doi:10.1021/ac501436d

Lee, W., Lee, V., Polio, S., Keegan, P., Lee, J.-H., Fischer, K., et al. (2010). On-demand three-dimensional freeform fabrication of multi-layered hydrogel scaffold with fluidic channels. Biotechnol. Bioeng. 105, 1178. doi:10.10.1002/bit.22613

Matter-Parrat, V., and Liverneaux, P. (2019). Impression 3D en chirurgie de la main. Hand Surg. Rehabilitat. doi:10.1016/j.hansur.2019.09.006

Melchels, F. P. W., Feijen, J., and Grijpma, D. W. (2010). A review on stereolithography and its applications in biomedical engineering. Biomaterials. 31 (24), 6121-6130. doi:10.1016/j.biomaterials.2010.04.050

Mohamed, O. A., Masood, S. H., and Bhowmik, J. L. (2015). Optimization of fused deposition modelling process parameters: a review of current research and future prospects. Adv. Manufacturing. 3 (1), 42-53. doi:10.1007/s40436-0140097-7

Molitch-Hou, M. (2018). Overview of additive manufacturing process. Add. Manuf. 2018, 1-38. doi:10.1016/b978-0-12-812155-9.000013

Mukherjee, P., Rani, A., and Saravanan, P. (2019). Polymeric materials for 3D bioprinting. 3D Print. Tech. Nanomedicine. 2019, 63-81. doi:10.1016/b978-012-815890-6.00004-9

Munir, K. S., Li, Y., and Wen, C. (2017). Metallic scaffolds manufactured by selective laser melting for biomedical applications. Metall. Foam Bone 1-23. doi:10.1016/b978-0-08-101289-5.00001-9

Muzaffar, A., Ahamed, M. B., Deshmukh, K., Kováŕík, T., Křenek, T., and Pasha, S. K. K. (2020). 3D and 4D printing of $\mathrm{pH}$-responsive and functional polymers and their composites. 3D and 4D Print. Polymer Nanocomp. Mat. 2020, 85-117. doi:10.1016/b978-0-12-816805-9.00004-1

Oh, S., and Choi, S. (2018). 3D-Printed capillary circuits for calibration-free viscosity measurement of Newtonian and Non-Newtonian fluids. Micromachines 9 (7), 314. doi:10.3390/mi9070314

Papaioannou, T. G., Manolesou, D., Dimakakos, E., Tsoucalas, G., Vavuranakis, M., and Tousoulis, D. (2019). 3D bioprinting methods and techniques: applications on artificial blood vessel fabrication. Acta Cardiol. Sin. 35 (3), 284. doi:10.6515/ACS.201905_3510.6515/ACS.201905_35(3).20181115A

Park, C., Lee, J., Kim, Y., Kim, J., Lee, J., and Park, S. (2017). 3D-printed microfluidic magnetic preconcentrator for the detection of bacterial pathogen using an ATP luminometer and antibody-conjugated magnetic nanoparticles. J. Microbiol. Meth. 132, 128-133. doi:10.1016/j.mimet.2016.12.001

Ragones, H., Schreiber, D., Inberg, A., Berkh, O., Kosa, G., Freeman, A., et al. (2015). Disposable electrochemical sensor prepared using 3D printing for cell and tissue diagnostics. Sensors Actuators B: Chem. 216, 434-442. doi:10.1016/j. snb.2105.04.065

Roda, A., Guardigi, M., Calabria, D., Calabretta, M. M., Cevenini, L., and Michelini, E. (2014). A 3D-printed device for a smartphone-based chemiluminescence biosensor for lactate in oral fluid and sweat. Analyst. 139 (24), 6494-6501. doi:10.1039/c4an01612b

Ruiz, C., Kadimisetty, K., Yin, K., Mauk, M. G., Zhao, H., and Liu, C. (2020). Fabrication of hard-soft microfluidic devices using hybrid 3D printing. Micromachines. 11 (6), 567. doi:10.3390/mi11060567

Salonitis, K. (2014). "Stereolithography," in Comprehensive materials processing. Amsterdam, Netherlands: Elsevier, 19-67. doi:10.1016/b978-0-08-096532-1.01001-3

Samper, I. C., Gowers, S. A. N., Rogers, M. L., Murray, D. R. K., Jewell, S. L., Pahl, C., et al. (2019). 3D printed microfluidic device or online detection of neurochemical changes with high temporal resolution in human brain microdialysate. R. Soc. Chem. Lab Chip. 19, 2038-2048. doi:10.1039/c9lc00044e

Santangelo, M. F., Libertino, S., Turner, A. P. F., Filippini, D., and Mark, W. C. (2017). Integrating printed microfluidics with silicon photomultipliers for miniaturised and highly sensitive ATP bioluminescence detection. Biosens. Bioelectron. 99, 464-470. doi:10.1016/j.bos.2017.07.05510.1016/j.bios.2017.07.055

Schönberger, M., and Hoffstetter, M. (2016). Generative manufacturing technologies-the future? Emerging Trends Med. Plast. Eng. Manufacturing. 2016, 107-174. doi:10.1016/b978-0-323-37023-3.00004-x

Sibbitt, J. P., and He, M. (2017). 3D printing of microfluidics for point of care diagnosis. Bio Sustain. Manufacturing. 2017, 2778. doi:10.1115/msec2017-2778 
Singh, R., Singh, S., and Hashmi, M. S. J. (2016). "Implant materials and their processing techanologies," in Reference module in materials science and materials engineering. doi:10.1016/b978-0-12-8.04156-4

Su, C. K., Yen, S. C., Li, T. W., and Sun, Y. C. (2016). Enzyme-immobilized 3Dprinted reactors for online monitoring of rat brain extracellular glucose and lactate. Anal. Chem. 88 (12), 6265-6273. doi:10.1021/acs.analchem.6b00272

Sweet, E., Yang, B., Chen, J., Vickerman, R., Lin, Y., Long, A., et al. (2020). 3D microfluidic gradient generator for combination antimicrobial susceptibility testing. Microsys. Nanoeng. 6, 92. doi:10.1038/s41378-020-00200-7

Symes, M. D., Kitson, P. J., Yan, J., Richmond, C. J., Cooper, G. J. T., Bowman, R. W., et al. (2012). Integrated 3D-printed reactionware for chemical synthesis and analysis. Nat. Chem. 4, 349. doi:10.1038/NCHEM.1313

Tang, C. K., Vaze, A., and Rusling, J. F. (2017). Automated 3D-printed unibody immunoarray for chemiluminescence detection of cancer biomarker proteins. Lab Chip. 17 (3), 484-489. doi:10.1039/c6lc01238h

Tappa, K., and Jammalamadaka, U. (2018). Novel biomaterials used in medical 3d printing techniques. J. Funct. Biomater. 9 (1), 17. doi:10.3390/jfb9010017

Tarn, M., and Nicole, P. (2013). Microfluidics. Mol. Sci. Chem. Eng. 2013, 1-7. doi:10.1016/b978-0-12-409547-2.05351-8

Wang, S., Ge, L., Yan, M., Yu, J., Song, X., Ge, S., et al. (2013). 3D microfluidic origami electrochemiluminescence immunodevice for sensitive point-of-care testing of carcinoma antigen 125. Sensors Actuators B: Chem. 176, 1-8. doi:10. 1016/j.snb.2012.08.035

Weisgrab, G., Ovsianikov, A., and Costa, P. F. (2019). Functional 3D printing for microfluidic chips. Adv. Mater. Technol. 4, 1900275. doi:10.1002/admt. 201900275
Wu, W., Deconinck, A., and Lewis, J. A. (2011). Omnidirectional printing of 3D microvascular networks. Adv. Mater. Weinheim. 23 (24), H178-H183. doi:10. 1002/adma.201004625

Yao, P., Xu, T., and Tung, S. (2018). Pneumatic microfluidic device by 3D printing technology for insulin determination. IEEE 12th Int. Conf. Nano/Molecular Med. Eng. (Nanomed). 2018, 8641565. doi:10.1109/nanomed.2018.8641565

Yeong, W. Y., Sudarmadji, N., Yu, H. Y., Chua, C. K., Leong, K. F., Venkatraman, S. S., et al. (2010). Porous polycaprolactone scaffold for cardiac tissue engineering fabricated by selective laser sintering. Acta Biomater. 6 (6), 2028-2034. doi:10. 1016/j.actbio.2009.12.033

Zargaryan, A., Farhoudi, N., Haworth, G., Ashby, J. F., and Au, S. H. (2020). Hybrid 3D printed-paper microfluidics. Nat. Res. 10, 18379. doi:10.1038/s41598-020-75489-5

Zhang, Y. (2019). Three-dimensional-printing for microfluidics or the other way around? Int. J. Bioprint. 5 (2), 192. doi:10.18063/ijb.v5i2.192

Conflict of Interest: The authors declare that the research was conducted in the absence of any commercial or financial relationships that could be construed as a potential conflict of interest.

Copyright (c) 2021 Prabhakar, Sen, Dwivedi, Khan, Solanki, Srivastava and Dhand This is an open-access article distributed under the terms of the Creative Commons Attribution License (CC BY). The use, distribution or reproduction in other forums is permitted, provided the original author(s) and the copyright owner(s) are credited and that the original publication in this journal is cited, in accordance with accepted academic practice. No use, distribution or reproduction is permitted which does not comply with these terms. 